\title{
High-Speed Stark Wavelength Tuning of MidIR Interband Cascade Lasers
}

\author{
Sergey Suchalkin, Mikhail V. Kisin, Serge Luryi, Gregory Belenky, Fred J. Towner, John D. Bruno, and \\ Richard L. Tober
}

\begin{abstract}
Stark modulation of the wavelength of a midinfrared tunable interband cascade laser was studied. Wavelength modulation at frequencies exceeding $1 \mathrm{GHz}$ was demonstrated. Estimates of capacitances inherent to the laser's structure suggested that improved packaging techniques could lead to modulation frequencies approaching $6 \mathrm{GHz}$.
\end{abstract}

Index Terms-Cascade lasers, midinfrared (midIR) lasers, semiconductor lasers, tunable lasers.

$\mathbf{T}$ UNABLE semiconductor lasers can be used in a wide variety of applications ranging from remote sensing and environmental monitoring to free space communications. The extent of their utility depends on how rapidly they can be modulated. Recent development of ultrafast infrared quantum-well photodetectors and optical heterodyning technique made possible to extent the detection bandwidth to $40 \mathrm{GHz}$ [1, and the references therein]. Lasers that rely on temperature tuning depend on the heat exchanged between the laser and surrounding media and, to a large extent, by the thermal capacitance of the laser which is proportional to the size of the device. Despite the relatively slow thermal processes involved, small single-mode InP-based tunable vertical-cavity surface-emitting lasers have been demonstrated at frequencies as high as $\sim 5 \mathrm{MHz}$ [2]. However, applications such as fast process control or optical FM communication [3] require much faster tuning methods.

Interband cascade lasers (ICLs) [4] are the devices operating in the midinfrared (midIR) spectral range $(3-5 \mu \mathrm{m})$, whose design combines interband optical transition and cascaded injection. The large Stark wavelength shift recently demonstrated in ICLs [5] results from inherently fast processes and thus shows promise for applications requiring gigahertz modulation frequencies. The goals of this letter are to demonstrate ultrafast Stark tuning of an ICL's wavelength and to discuss factors that limit its modulation bandwidth.

The molecular beam epitaxy-grown tunable ICL used in these experiments has 14 periods, each having a digitally graded

Manuscript received November 22, 2006; revised January 3, 2007. Work at SUNY was supported by ARO grant W911NF0610399 and by NYSTAR under Contract C020000. The work of F. J. Towner and J. D. Bruno were supported by MDA under AFRL Contract F19628-02-C-0032 and by DOE under Contract DE-FG02-02ER83492.

S. Suchalkin, M. V. Kisin, S. Luryi, and G. Belenky are with the Department of Electrical and Computer Engineering, SUNY at Stony Brook, Stony Brook, NY 11794 USA (e-mail: suchal@ece.sunysb.edu).

F. J. Towner and J. D. Bruno are with Maxion Technologies Inc., Hyattsville, MD 20782 USA.

R. L. Tober is with the Army Research Laboratory, Adelphi, MD 20873 USA. Digital Object Identifier 10.1109/LPT.2007.891649

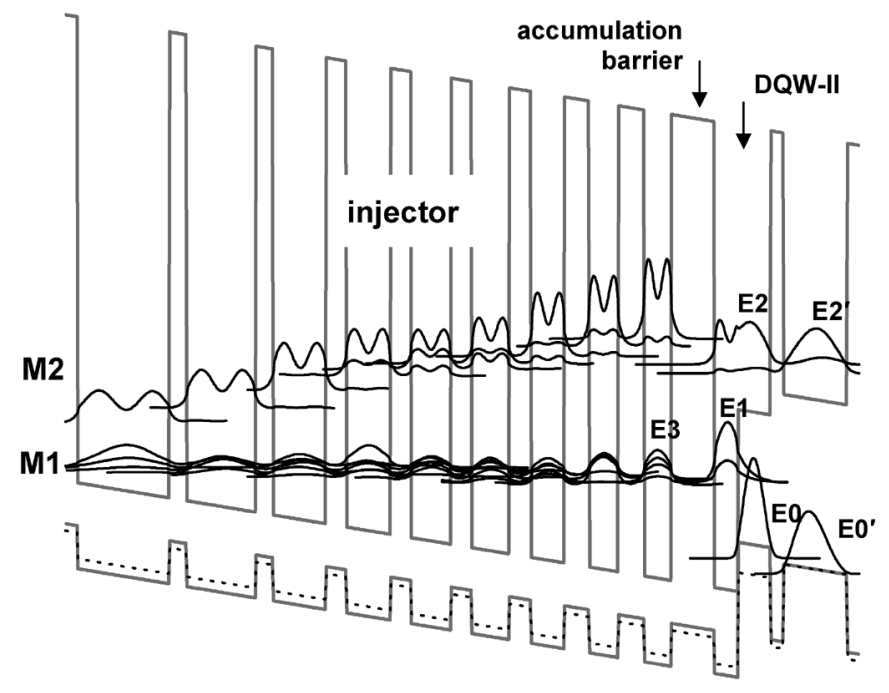

Fig. 1. Band diagram of a single period of the tunable ICL structure under external electric field $70 \mathrm{kV} / \mathrm{cm}$. Lower (E0) and upper (E1) lasing states are located in the optically active type-II DQW structure (DQW-II). Electron injection through the manifold $\mathrm{M} 1$ of the injector states is accompanied by electron accumulation in the injector quantum well (E3) adjacent to the accumulation barrier.

InAs-AlSb injector region and $\mathrm{InAs}-\mathrm{Ga}_{0.8} \mathrm{In}_{0.2} \mathrm{Sb}-\mathrm{GaSb}$ type-II double quantum-well (DQW) heterostructure separated from the injector by a 4-nm AlSb accumulation barrier. (A detailed description of the laser structure can be found in [5].) The laser device was processed into a $35 \mu \mathrm{m} \times 0.5 \mathrm{~mm}$ and $8 \mu \mathrm{m} \times 0.5 \mathrm{~mm}$ ridge waveguide and indium soldered, epi-up, onto a Au-coated copper mount. The mounts were then attached to the cold finger of a liquid nitrogen cryostat for experiments performed at $80 \mathrm{~K}$ and to the cold finger of a closed cycle cooler for experiments performed at $60 \mathrm{~K}$.

The band structure and eigenstate probability distributions in an external electric field of $70 \mathrm{kV} / \mathrm{cm}$ are shown in Fig. 1. They were calculated using an eight-band $\mathrm{k} \bullet \mathrm{p}$ model including strain-related effects. The mini-band labeled with M1 illustrates how the lower levels in the quantum wells composing the injector region align to insure that electrons transport into the accumulation energy state $E_{3}$ and then tunnel through the accumulation barrier into the upper lasing level $E_{1}$ of the type-II active DQW. The electrons eventually relax into the lower lasing level $E_{0}$ with the emission of a photon. The upper manifold, $\mathrm{M} 2$, has a series of misaligned states localized in each of the injector region quantum wells which align at higher electric fields $(\sim 110 \mathrm{kV} / \mathrm{cm})$.

The barrier separating the injector from the type-II DQW was designed so that electrons would accumulate at the edge of the 


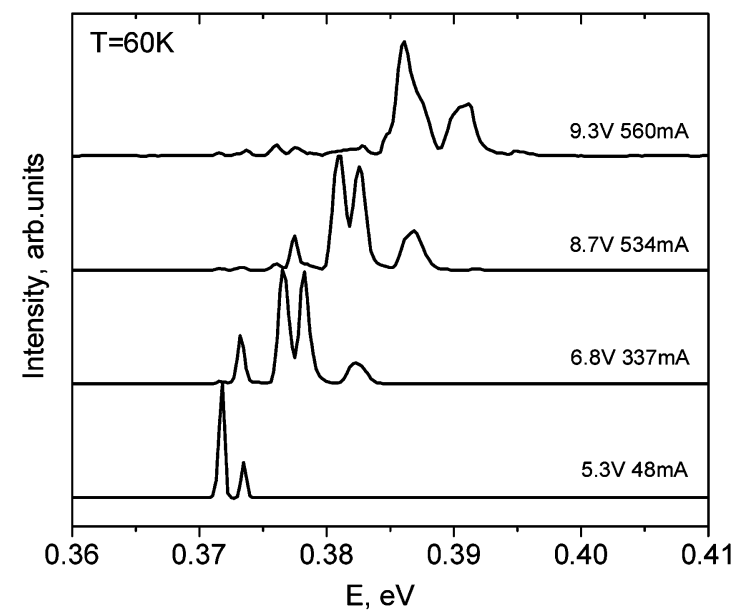

Fig. 2. Lasing spectra of the tunable ICL at different bias currents.

injection region at $E_{3}$ before tunneling into $E_{1}$. Holes must simultaneously accumulate in the lower (hole) lasing level $E_{0}$ in the type-II DQW in order to maintain overall charge neutrality. This creates a charge separation and a corresponding electric field (perpendicular to the epi-layers) that increases with the injection current. Thus, the energy separation between $E_{1}$ and $E_{0}$ (and hence the emission wavelength) undergoes a linear Stark shift that depends on the bias current which controls the electric field in the optically active region. That is, the charge carrier accumulation outside of the optically active layers affects the laser wavelength even above the laser threshold [5].

As the bias current increases, the modal gain spectrum of the laser shifts continuously to the high energy side due to the Stark effect. The emission spectrum shifts toward shorter wavelengths as well but it discontinuously hops between longitudinal modes (i.e., separated by $\sim 3 \mathrm{~cm}^{-1}$ or $0.4 \mathrm{meV}$ as expected from the cavity length) that occur under the envelope of the modal gain spectrum. In addition to the longitudinal mode hopping, consecutive switching between separate mode groups is observed at increasing bias current (Fig. 2). The laser lines shown in Fig. 2 are measured at a 35-mm mesa device. The peaks are longitudinal mode groups; the fine structure of the spectra is not shown in the figure. The effect of mode grouping is attributed to the formation of substrate "leaky" modes [5]. Leaky modes are formed as a result of penetration of the optical field through the lower cladding layer and interference in the substrate. The shift increment due to the leaky modes $\left(\sim 32 \mathrm{~cm}^{-1}\right.$ or $\left.4 \mathrm{meV}\right)$ is determined by the effective modal indices, geometrical parameters of the laser cavity, and the substrate thickness. The effect of mode grouping will be used here to demonstrate the high speed of the observed Stark tuning.

To obtain experimental information about the tuning speed of the device, we applied combined a dc and ac bias to $8-\mu \mathrm{m}$ mesa device. The ac component was a harmonic bias modulation within the frequency range 10-2000 MHz. The dc bias current was $31 \mathrm{~mA}$; the ac modulation amplitude was $\sim 25 \mathrm{~mA}$. Both components were decoupled from each other with a high bandwidth $(4.2 \mathrm{GHz})$ bias-tee. The spectrum of the laser was analyzed with a Fourier transform infrared spectrometer equipped with an InSb photovoltaic detector. Since the response

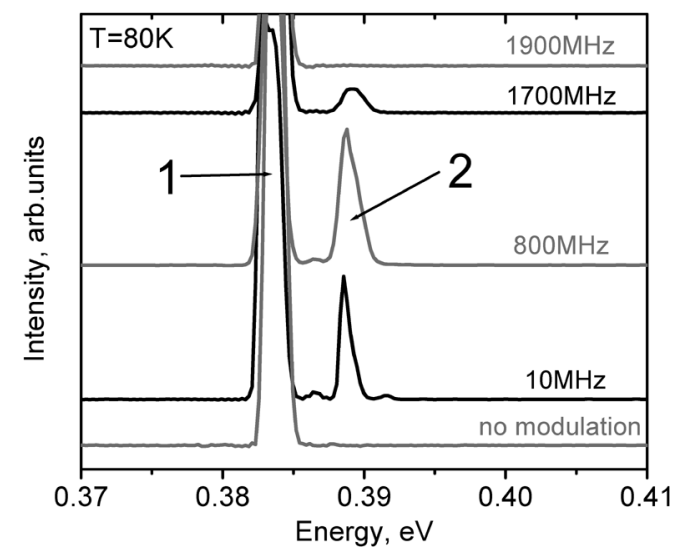

Fig. 3. Lasing spectra of the tunable ICL at different bias modulation frequencies.

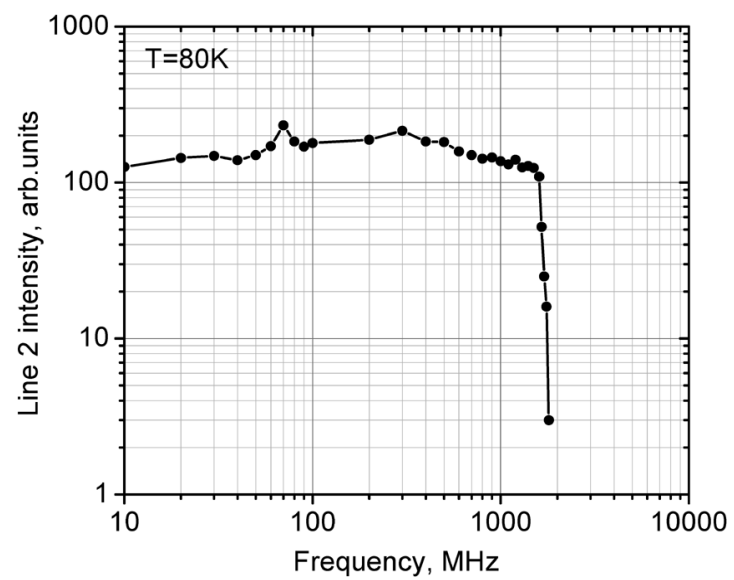

Fig. 4. Dependence of the intensity of the Line 2 on bias modulation frequency.

time of the detector $\tau$ exceeded $10^{-7} \mathrm{~s}$, only time-averaged spectra were measured. The differential resistance of the laser with the mesa size $8 \times 500 \mu \mathrm{m}$ at the given dc bias was $6.9 \Omega$. A high-frequency $47-\Omega$ resistor was connected in series with the laser to improve the cable impedance matching. For the $31-\mathrm{mA}$ bias, the laser spectrum (Fig. 3) consists of a single group of longitudinal modes (further referred to as Line 1). As the bias current increases to $\sim 40 \mathrm{~mA}$, another mode group (Line 2) corresponding to the next "leaky mode maximum" appears due to the Stark shift of the gain spectrum. The presence of Line 2 in the time-averaged spectrum means that the tuning response time is shorter than the laser modulation period. At modulation frequencies above 1.6-GHz, Line 2 starts to disappear, and at higher frequencies and the laser spectrum shape returns to that without modulation. The relative amplitude of the second line as a function of bias modulation frequency (Fig. 4) allows for a rough estimation of the wavelength modulation bandwidth. The sharp decrease of the intensity of Line 2 takes place at $\sim 1.6 \mathrm{GHz}$. The disappearance of Line 2 means that the peak bias current has decreased from 56 to $\sim 40 \mathrm{~mA}$ so that the modulation amplitude decreased $\sim 2.5$ times.

One of the factors limiting the wavelength tuning bandwidth is the $R C$ time of the device determined by the differential resistance and capacitance, $R$ and $C$. The total capacitance of the device $C$ is the sum of the laser structure capacitance $C_{L}$ and 
the capacitance of the laser package $C_{P}$. To estimate the differential capacitance of the tunable laser above threshold, we calculate it as 14 plane capacitors connected in series. Each capacitor represents a type-II quantum well sandwiched between electron and hole accumulation quantum wells. Taking $\varepsilon \sim 12$ and the distance between the "plates" to be $\sim 80 \AA$, we obtain $C_{L} \sim 4 \mathrm{pF}$. We neglected the capacitance of the injectors and claddings assuming that above threshold the additional charge accumulates mostly in the electron and hole accumulation wells, QW3 and QW1. The differential resistance of the laser is $\sim 7 \Omega$, so the wavelength modulation bandwidth of the ideal device can be estimated as $\Delta \nu \sim 1 / 2 \pi R C_{L} \sim 6 \mathrm{GHz}$. For a real device, the bandwidth is reduced by the parasitic capacitance of the package $C_{P}$. The main contribution to $C_{P}$ is provided by two bonding pads and the substrate separated by $0.3 \mu \mathrm{m}$ of silicon nitride. Taking $\varepsilon \sim 5$ for silicon nitride [7], we obtain $C_{P} \sim 15 \mathrm{pF}$ for two $100 \times 500 \mu \mathrm{m}$ bonding pads. This reduces the device modulation bandwidth to $\sim 1.2 \mathrm{GHz}$, which is close to the frequency at which the wavelength tuning collapses. It should be noted that the concentration pinning becomes "softer" as the frequency approaches the relaxation resonance. For such frequencies, the modulation of the electric field in the active area can be obtained even without an accumulation barrier. A typical relaxation resonance frequency for an in-plane laser usually exceeds a few gigahertz [8]. The known exception is the intersubband quantum cascade laser, where the relaxation resonance is suppressed [9]. The fact that results presented here demonstrate wavelength modulation at frequencies well below the relaxation resonance (i.e., $10 \mathrm{MHz}$ ) indicates that the ICL's tuning is indeed due to a Stark shift associated with the charge accumulated outside the optical active region.
In conclusion, we have demonstrated Stark modulation of the ICL wavelength at frequencies exceeding $1 \mathrm{GHz}$. This is at least two orders of magnitude greater than that achieved with the temperature tuning techniques. Moreover, estimates suggest that improved packaging could increase the maximum modulation frequency of the tunable ICLs to $\sim 6 \mathrm{GHz}$.

\section{REFERENCES}

[1] P. D. Grant, R. Dudeck, M. Buchanan, L. Wolfson, and H. C. Liu, "An ultra fast quantum well infrared photodetector," Infrared Phys. Technol., vol. 47, pp. 144-152, 2005.

[2] M. Lakner, G. Totsching, F. Winter, M. Ortsiefer, M.-C. Amann, R. Shau, and J. Rosskopf, "Demonstration of methane spectroscopy using a vertical-cavity surface-emitting laser at $1.68 \mathrm{~mm}$ with up to $5 \mathrm{MHz}$ repetition rate," Meas. Sci. Technol., vol. 14, pp. 101-106, 2003.

[3] S. Luryi and M. Gouzman, "Feasibility of an optical frequency modulation system for free-space optical communications," Int. J. High Speed Electron. Syst., vol. 16, pp. 559-566, 2006.

[4] R. Q. Yang, J. D. Bruno, J. L. Bradshaw, J. T. Pham, and D. E. Wortman, "Interband cascade lasers: Progress and challenges," Physica E, vol. 7, p. 69, 2000.

[5] S. Suchalkin, M. V. Kisin, S. Luryi, G. Belenky, J. Bruno, F. J. Towner, and R. Tober, "Widely tunable type-II interband cascade laser," Appl. Phys. Lett., vol. 88, no. 3, 2006, Article 031103.

[6] D. Westerfeld, S. Suchalkin, M. Kisin, G. Belenky, J. Bruno, and R. Tober, "Experimental Study of optical gain and loss in 3.4-3.6 mm interband cascade lasers," Proc. Inst. Elect. Eng., Optoelectron., vol. 150, pp. 293-297, 2003.

[7] S. Fudjita, T. Ohishi, H. Toyoshima, and A. Sasaki, "Electrical properties of silicon nitride thin films plasma deposited from SiF4, N2 and H2 source gases," J. Appl. Phys., vol. 57, pp. 426-431, 1985.

[8] L. A. Coldren and S. W. Corzine, Diode Lasers and Photonic Integration Circuits. New York: Wiley, 1995.

[9] R. Paiella, R. Martini, F. Capasso, C. Gmahl, H. Y. Hwang, D. L. Sivco, J. N. Baillargeon, and A. Cho, "High frequency modulation without the relaxation oscillation resonance in quantum cascade lasers," Appl. Phys. Lett., vol. 79, pp. 2526-2528, 2001. 